\title{
Grid search of exponential smoothing method: a case study of Ho Chi Minh City load demand
}

\author{
Tran Thanh Ngoc, Le Van Dai \\ Faculty of Electrical Engineering Technology, Industrial University of Ho Chi Minh City, Vietnam
}

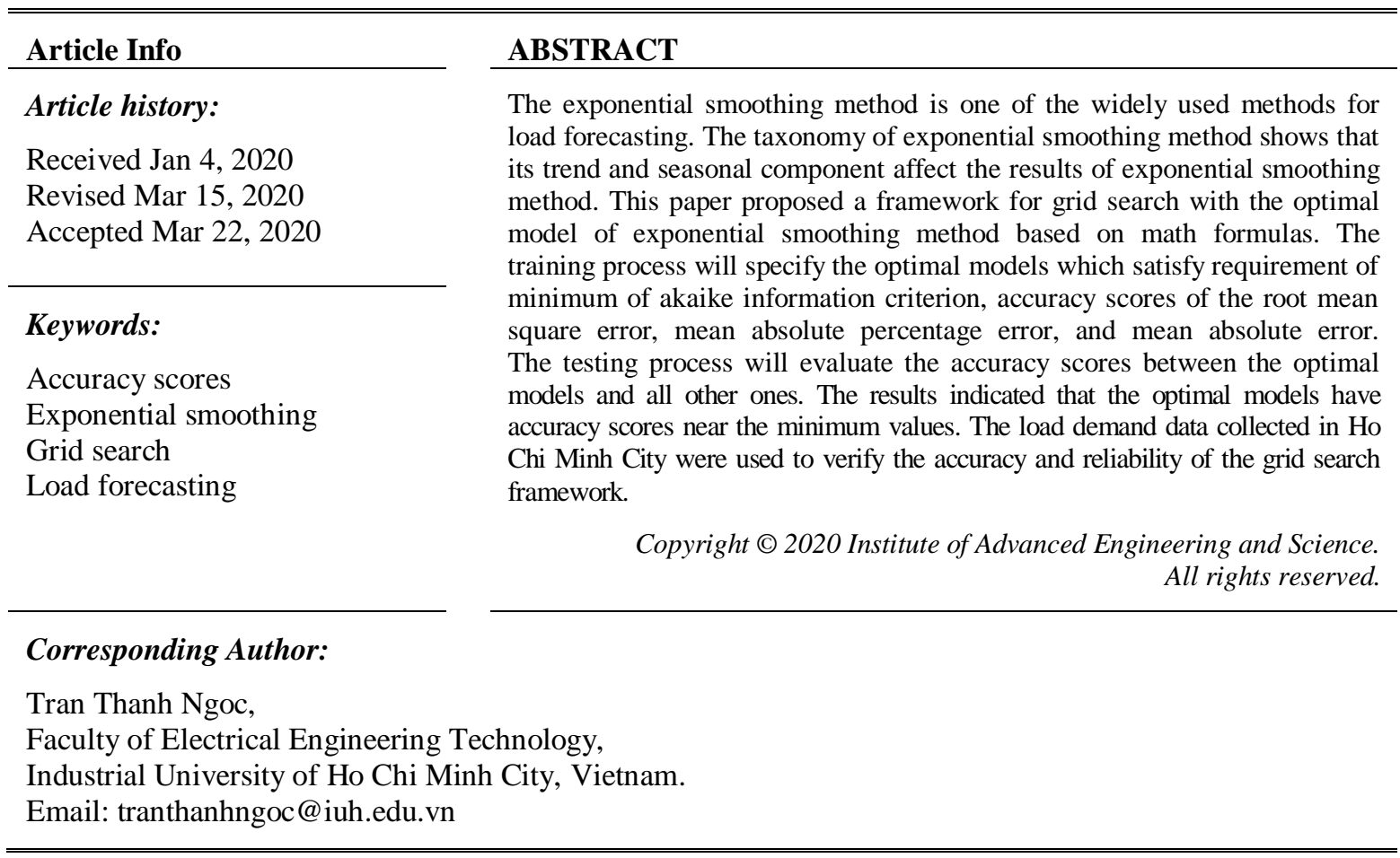

\section{INTRODUCTION}

Load demand forecasting is of great importance for electric power systems operation, transmission, distribution and retail of electricity. According to different horizons, load forecasting can be classified into three categories: short-term load forecasting (STLF), medium-term forecasting (MTLF) and long-term forecasting (LTLF). STLF is usually utilized from one hour to a week toward hourly, daily or weekly system energy, while MTLF is usually from a week to a year and LTLF is more than a year [1-6].

Among several techniques used for load forecasting such as Multiple Regression, Exponential Smoothing, Stochastic Time Series, Fuzzy Logic, Neural Networks and Knowledge-Based Expert Systems; exponential smoothing (ES) method has known as one of the most promising forecasting strategies due to its simplicity, robustness and accuracy [7-10]. The ES method assumes that the time series consist of three components: level, trend and seasonality. The trend component can include five types: none, additive, damped additive, multiplicative, or damped multiplicative; and the seasonality can include three types: none, additive, or multiplicative. Thus, the taxonomy of ES method consists of 15 different ES models. Many of them are well known, including simple exponential smoothing (trend: none, seasonality: none), Holt's linear model (trend: additive, seasonality: none), Additive Holt-Winters' model (trend: additive, seasonality: additive), multiplicative Holt-Winters' model (trend: additive, seasonality: multiplicative) [8, 11-13].

Recently, there have been many studies devoted to clarifying advantages of ES method as well as applying it for load forecasting [14-26]. The main point that should be noticed here is that the researchers commonly used only one specific ES model of 'Additive Holt-Winters' or 'Multiplicative Holt-Winters' as a default type of ES method, while there could be other approaches that could exist and the utilization of which can give better results. In this regard, the present work aims to propose a framework for Grid search using the optimal model of ES method based on the math formulas of ES taxonomy. The optimal model will be 
evaluated in the testing process based on accuracy scores such as the root mean square error (RMSE), mean absolute percentage error (MAPE), and mean absolute error (MAE). The load demand data of Ho Chi Minh City was used to analyze by splitting them into in many cases to consolidate the obtained results.

\section{TAXONOMY OF ES METHOD}

The ES method assumes that the time series consist of three underlying data components: level, trend, and seasonality. The trend of ES method can include one of five types of trend: none, additive, multiplicative, damped additive, and damped multiplicative. While the seasonality of ES method can include one of three types: none, additive, and multiplicative. By combining the trend and seasonal components, 15 different ES models could be established as listed in Table 1 [8, 11-13]. Table 2 shows the math formulas for 15 ES models based on Table 1. Each cell consists of smoothing equation for applying the method and the forecast equation.

Table 1. A Taxonomy of ES method

\begin{tabular}{cccc}
\hline Trend Component & \multicolumn{3}{c}{ Seasonal Component } \\
\hline & N (None) & A (Additive) & M (Multiplicative) \\
\hline N (None) & N,N & N,A & N,M \\
A (Additive) & A,N & A,A & A,M \\
Ad (Additive damped) & Ad,N & Ad,A & Ad,M \\
M (Multiplicative) & M,N & M,A & M,M \\
Md (Multiplicative damped) & Md,N & Md,A & Md,M \\
\hline
\end{tabular}

Table 2. A Taxonomy of ES method: formulas

\begin{tabular}{|c|c|c|c|}
\hline \multirow{2}{*}{ Trend } & \multicolumn{3}{|c|}{ Seasonal } \\
\hline & $\mathrm{N}$ & A & M \\
\hline $\mathrm{N}$ & $\begin{array}{c}\hat{y}_{t+h \mid t}=l_{t} \\
l_{t}=\alpha y_{t}+(1-\alpha) l_{t-1}\end{array}$ & $\begin{array}{c}\hat{y}_{t+h \mid t}=l_{t}+s_{t+h-m(k+1)} \\
l_{t}=\alpha\left(y_{t}-s_{t-m}\right)+(1-\alpha) l_{t-1} \\
s_{t}=\gamma\left(y_{t}-l_{t-1}\right)+(1-\gamma) s_{t-m}\end{array}$ & $\begin{array}{c}\hat{y}_{t+h \mid t}=l_{t} s_{t+h-m(k+1)} \\
l_{t}=\alpha\left(y_{t} / s_{t-m}\right)+(1-\alpha) l_{t-1} \\
s_{t}=\gamma\left(y_{t} / l_{t-1}\right)+(1-\gamma) s_{t-m}\end{array}$ \\
\hline A & $\begin{array}{c}\hat{y}_{t+h \mid t}=l_{t}+h b_{t} \\
l_{t}=\alpha y_{t}+(1-\alpha)\left(l_{t-1}+b_{t-1}\right) \\
b_{t}=\beta\left(l_{t}-l_{t-1}\right)+(1-\beta) b_{t-1}\end{array}$ & $\begin{array}{l}\hat{y}_{t+h \mid t}=l_{t}+h b_{t}+s_{t+h-m(k+1)} \\
l_{t}=\alpha\left(y_{t}-s_{t-m}\right)+(1-\alpha)\left(l_{t-1}+b_{t-1}\right) \\
b_{t}=\beta\left(l_{t}-l_{t-1}\right)+(1-\beta) b_{t-1} \\
s_{t}=\gamma\left(y_{t}-l_{t-1}-b_{t-1}\right)+(1-\gamma) s_{t-m} \\
\hat{y}_{t+h \mid t}=l_{t}+\phi_{h} b_{t}+s_{t+h-m}\end{array}$ & $\begin{array}{l}\hat{y}_{t+h \mid t}=\left(l_{t}+h b_{t}\right) s_{t+h-m(k+1)} \\
l_{t}=\alpha\left(y_{t} / s_{t-m}\right)+(1-\alpha)\left(l_{t-1}+b_{t-1}\right) \\
b_{t}=\beta\left(l_{t}-l_{t-1}\right)+(1-\beta) b_{t-1} \\
s_{t}=\gamma\left(y_{t} /\left(l_{t-1}+b_{t-1}\right)\right)+(1-\gamma) s_{t-m} \\
\hat{y}_{t+h l t}=\left(l_{t}+\phi_{h} b_{t}\right) s_{t+h-m}\end{array}$ \\
\hline$A_{d}$ & $\begin{array}{c}\hat{y}_{t+h \mid t}=l_{t}+\phi_{h} b_{t} \\
l_{t}=\alpha y_{t}+(1-\alpha)\left(l_{t-1}+\phi b_{t-1}\right) \\
b_{t}=\beta\left(l_{t}-l_{t-1}\right)+(1-\beta) \phi b_{t-1}\end{array}$ & $\begin{array}{c}c_{t}=\alpha\left(y_{t}-s_{t-m}\right)+(1-\alpha)\left(l_{t-1}\right. \\
\left.+\phi b_{t-1}\right) \\
b_{t}=\beta\left(l_{t}-l_{t-1}\right)+(1-\beta) \phi b_{t-1} \\
s_{t}=\gamma\left(y_{t}-l_{t-1}-\phi b_{t-1}\right)+(1-\gamma) s_{t-m}\end{array}$ & $\begin{array}{c}l_{t}=\alpha\left(y_{t} / s_{t-m}\right)+(1-\alpha)\left(l_{t-1}\right. \\
\left.\quad+\phi b_{t-1}\right) \\
b_{t}=\beta\left(l_{t}-l_{t-1}\right)+(1-\beta) \phi b_{t-1} \\
s_{t}=\gamma\left(y_{t} /\left(l_{t-1}+\phi b_{t-1}\right)\right)+(1-\gamma) s_{t-m}\end{array}$ \\
\hline M & $\begin{array}{l}\hat{y}_{t+h \mid t}=l_{t} b_{t}^{h} \\
l_{t}=\alpha y_{t}+(1-\alpha) l_{t-1} b_{t-1} \\
b_{t}=\beta\left(l_{t} / l_{t-1}\right)+(1-\beta) b_{t-1}\end{array}$ & $\begin{array}{l}\hat{y}_{t+h \mid t}=l_{t} b_{t}^{h}+s_{t+h-m(k+1)} \\
l_{t}=\alpha\left(y_{t}-s_{t-m}\right)+(1-\alpha) l_{t-1} b_{t-1} \\
b_{t}=\beta\left(l_{t} / l_{t-1}\right)+(1-\beta) b_{t-1} \\
\quad s_{t}=\gamma\left(y_{t}-l_{t-1} b_{t-1}\right)+(1-\gamma) s_{t-m}\end{array}$ & $\begin{array}{l}\hat{y}_{t+h \mid t}=l_{t} b_{t}^{h} s_{t+h-m(k+1)} \\
l_{t}=\alpha\left(y_{t} / s_{t-m}\right)+(1-\alpha) l_{t-1} b_{t-1} \\
b_{t}=\beta\left(l_{t} / l_{t-1}\right)+(1-\beta) b_{t-1} \\
\quad s_{t}=\gamma\left(y_{t} /\left(l_{t-1} b_{t-1}\right)\right)+(1-\gamma) s_{t-m}\end{array}$ \\
\hline $\mathrm{M}_{\mathrm{d}}$ & $\begin{array}{l}\hat{y}_{t+h \mid t}=l_{t} b_{t}^{\phi_{h}} \\
l_{t}=\alpha y_{t}+(1-\alpha) l_{t-1} b_{t-1}^{\phi} \\
b_{t}=\beta\left(l_{t} / l_{t-1}\right)+(1-\beta) b_{t-1}^{\phi}\end{array}$ & $\begin{array}{l}\hat{y}_{t+h \mid t}=l_{t} b_{t}^{\phi_{h}}+s_{t+h-m(k+1)} \\
l_{t}=\alpha\left(y_{t}-s_{t-m}\right)+(1-\alpha) l_{t-1} b_{t-1}^{\phi} \\
b_{t}=\beta\left(l_{t} / l_{t-1}\right)+(1-\beta) b_{t-1}^{\phi} \\
s_{t}=\gamma\left(y_{t}-l_{t-1} b_{t-1}^{\phi}\right)+(1-\gamma) s_{t-m}\end{array}$ & $\begin{array}{l}\hat{y}_{t+h \mid t}=l_{t} b_{t}^{\phi} s_{t+h-m(k+1)} \\
l_{t}=\alpha\left(y_{t} / s_{t-m}\right)+(1-\alpha) l_{t-1} b_{t-1}^{\phi} \\
b_{t}=\beta\left(l_{t} / l_{t-1}\right)+(1-\beta) b_{t-1}^{\phi} \\
s_{t}=\gamma\left(y_{t} /\left(l_{t-1} b_{t-1}^{\phi}\right)\right)+(1-\gamma) s_{t-m}\end{array}$ \\
\hline
\end{tabular}

where $l_{t}, b_{\mathrm{t}}$ and $s_{t}$ are the series level, the series trend and the series seasonal at time $t$, respectively; $m$ is the seasonal period; $\alpha, \beta, \gamma$ and $\phi$ are smoothing parameters, $\emptyset_{h}=\varnothing+\emptyset^{2}+\cdots+\emptyset^{h} ; h$ is the step ahead forecasts; $k$ is the integer part of $(h-1) / m$.

\section{GRID SEARCH FOR ES METHOD}

The first point that must be mentioned here is how to compare ES models. When a model is defined as one of the 15 ES models as listed in Table 2 above, the level smoothing coefficient $\alpha$, the trend smoothing coefficient $\beta$, the seasonal smoothing coefficient $\gamma$ and the damped trend coefficient $\phi$ can be specified by tools of some software as Python with stats models tool [27]. These tools will automatically tune and specify the optimal values for $\alpha, \beta, \gamma$ and $\phi$. There is more important and interesting problems, that how to specify the optimal structure of ES method. There are other hyper-parameters that will not be tuned automatically, and must be specified: 
a) The type of trend component: N, A, M

b) The type of damped component: True, False

c) The type of seasonal component: $\mathrm{N}, \mathrm{A}, \mathrm{M}$

d) The seasonal period m: 0; 4 (quarterly); 7 (weekly); 12 (monthly); 24 (hourly). Moreover, when using stats models tool in Python for ES method, we can specify the option for two other parameters:

e) The Box-Cox option (Apply the Box-Cox transform for the data first): True, False

f) The bias option (Remove bias from forecast values and fitted values by enforcing that the average residual is equal to zero): True, False

Thus, the configuration parameter of ES method consists of the trend type (t), the damped type (d), the seasonal type (s), the seasonal period type $(\mathrm{m})$, the Box-Cox transformer type (b), the remove bias (r) [13]. Next question is how to specify the optimal configuration of ES method for known data. The common method is to split the data into training data and testing data. The training process can specify the optimal model according the requirement of minimum error scores between the fitted values produced by the model and the training values. The test process can use testing data to evaluate reliability of the optimal model obtained from training process. There are many popular error scores for training process and testing process. In this paper, we will use the root mean square error (RMSE), mean absolute percent error (MAPE) and mean absolute error (MAE). These equations of RMSE, MAPE and MAE are given by [28-31]

$$
\begin{aligned}
& \text { RMSE }=\sqrt{\frac{1}{n} \sum_{i=1}^{n}\left|Y_{i}-F_{i}\right|^{2}} \\
& \text { MAPE }=\frac{1}{n} \sum_{i=1}^{n}\left|\frac{Y_{i}-F_{i}}{Y_{i}}\right| \\
& M A E=\frac{1}{n} \sum_{i=1}^{n}\left|Y_{i}-F_{i}\right|
\end{aligned}
$$

where $Y_{i}$ is the actual observed values, $F_{i}$ is the fitted or forecasting values, and $n$ is the number of observed values.

An alternative used to specify the optimal model in the training process is called as information criterion (IC). There are many ICs and the most common is akaike information criterion (AIC) which can be described by the following equation:

$$
A I C=-2 \log (L)+2 p
$$

where $p$ is the number of estimated parameters in the model and $L$ is the likelihood [19-20]. follows.

The framework for Grid search of ES method shown below in Figure 1 includes four steps as

Step 1: Split the data into training data $\left(\mathrm{y}_{\text {train }}\right)$ and testing data $\left(\mathrm{y}_{\text {test }}\right)$

Step 2: (i) Define the ES model based on combination of ( $t, d, s, p)$, note that the period of seasonal $p$ depends on the characteristic of each time series; (ii) Fit the model based on combination of (b, r); and (iii) Calculate the accuracy scores (RMSE, MAPE, and MAE) between the training values and the fitted values as well as obtain the Information criterion AIC.

Step 3: Calculate the accuracy scores (RMSE, MAPE, and MAE) between the testing values and the forecast values

Step 4: (i) Specify the optimal ES models in step 2 according to requirement of minimum the accuracy scores (RMSE, MAPE, MAE) and AIC, correspond; (ii) Analysis the optimal model and the other model based on the accuracy scores values of the test process; and (iii) Evaluate the grid search and give the conclusion. 


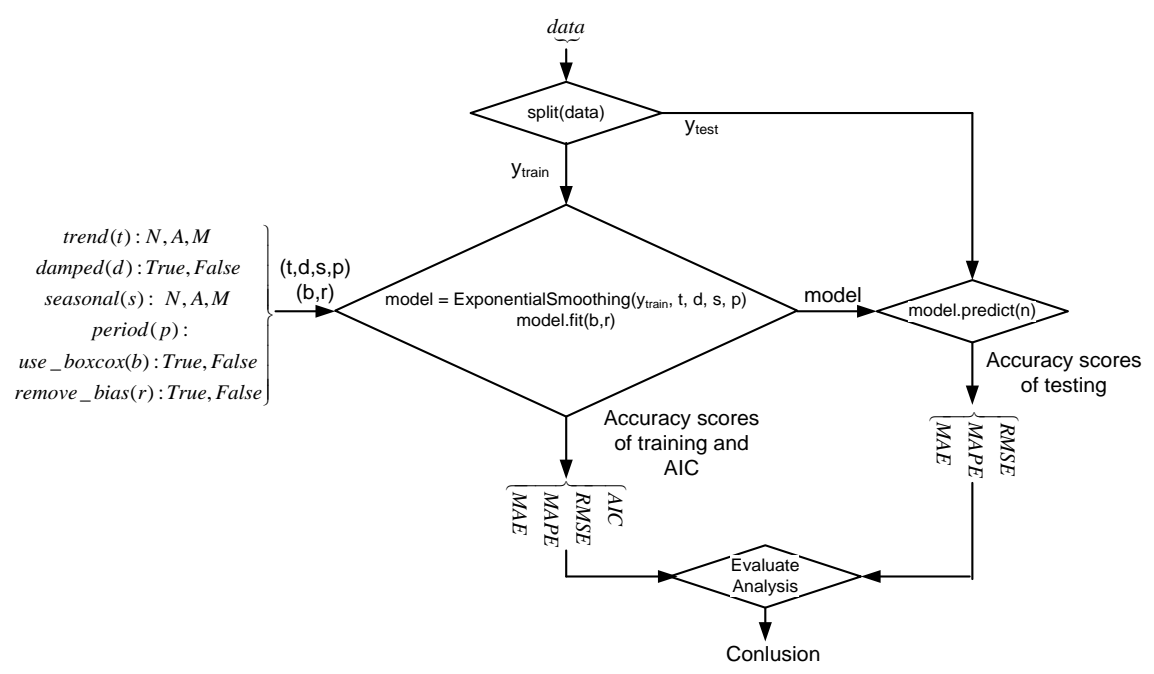

Figure 1. The framework for grid search of ES methods

\section{RESULTS AND ANALYSIS}

\subsection{Data description}

Ho Chi Minh City is the largest City in Vietnam, and is also one of Vietnam's most important economic, political, cultural and educational centers. With a population of over 10 million people and a high concentration of industrial clusters, Ho Chi Minh City's electricity is essential and always in great demand. In this paper, the one-hour load demand of Ho Chi Minh City has been applied in ES Grid search. Figures 2(a-d) show one-hour load demand for a typical year (from 2018-01-01 to 2018-12-30), a typical moth (from 2018-12-03 to 2018-12-30), a typical week (from 2018-12-24 to 2018-12-30) and a typical day (from 2018-12-30 00 to 2018-12-30 23), respectively.

As seen clearly in Figure 2, the seasonal cycle duration was obtained with $p=24$ and $p=168$ within one day and one week, respectively. Thus, in this paper, the seasonal period $\mathrm{p}$ is 0.24 , and 168 . In order to dismiss the random results, the data have been splitted into many cases as shown below in Table 3 , in which the testing data correspond to 1 month and 1 week, while the training data-1 year, 1 month and 1 week.

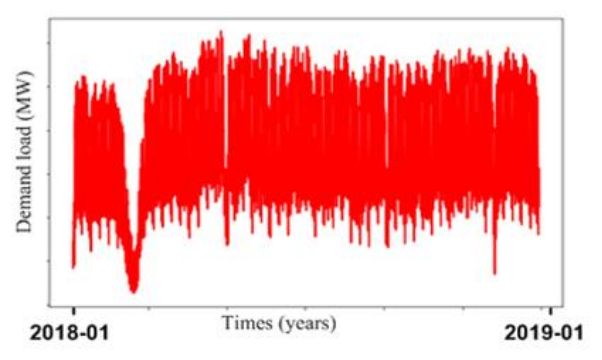

(a)

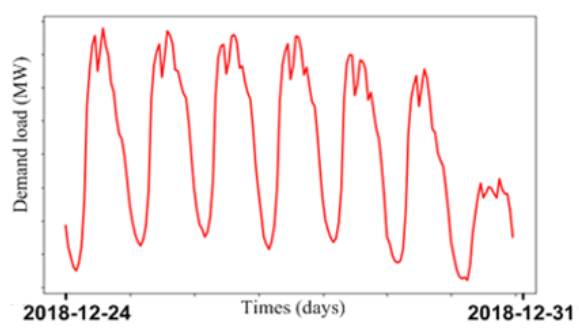

(c)



(b)

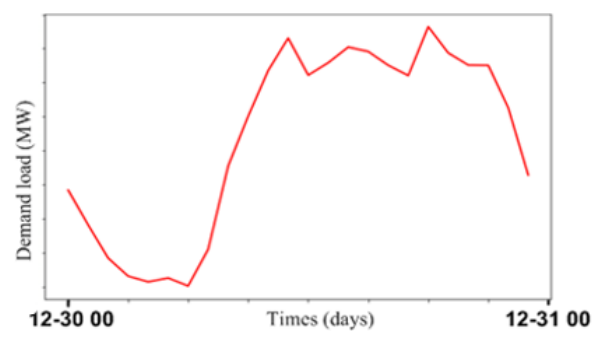

(d)

Figure 2. Electric demand in Ho Chi Minh city, Vietnam: (a) 01 year, (b) 01 month, (c) 01 week, (d) 1 day 
Table 3. The training and testing data

\begin{tabular}{ccc}
\hline Case & Training & Testing \\
\hline 1 & 1 month & 1 month \\
2 & 1 year & 1 month \\
3 & 1 week & 1 week \\
4 & 1 month & 1 week \\
\hline
\end{tabular}

\subsection{Results of case 1}

In case 1 , the training data is for 01 month from 2018-11-05 to 2018-12-02, and the testing dat -01 month from 2018-12-03 to 2018-12-30. Table 4 shows the results of training process, the optimal ES models and the results of testing process. As obviously seen that the minimum of AIC and RMSE gives the same optimal ES model as for MAPE and MAE. Therefore, in case 1, there were two optimal models found. For testing process, the column 'Optimal' shows the accuracy scores for the optimal ES model, and the column 'Min' and 'Average'-the min and the average values for all the possible models that can be generated in training process. Figure 3(a) gives the fitted and training series of the training process, while Figure 3(b) the forecast and testing series of testing process for the first optimal model. Figure 4 indicates the accuracy scores for testing process, for that Figure 4(a) presents the box plot for RMSE component with the first column for all the possible models, the second column for the first optimal model and the third column for the second-optimal model. The same distributed data are plotted in Figure 4(b) and 4(c).

Table 4. The results of case 1

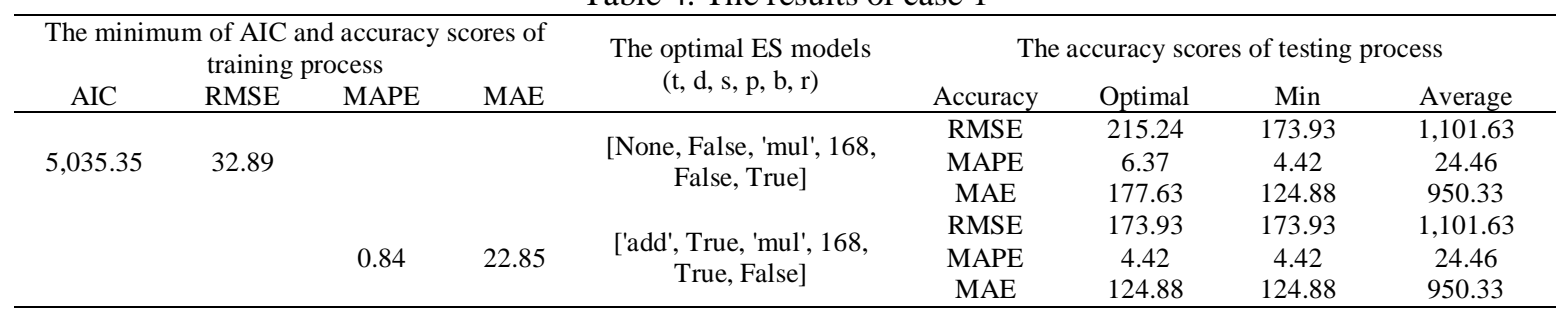

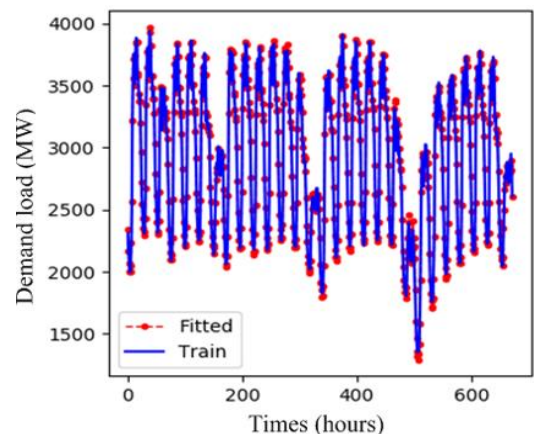

(a)

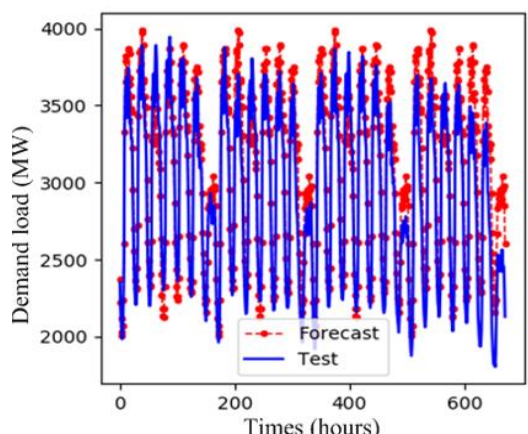

(b)

Figure 3. (a) Fitted and training series, (b) forecast and testing series

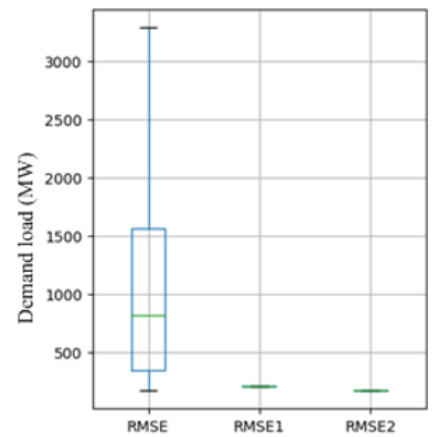

(a)

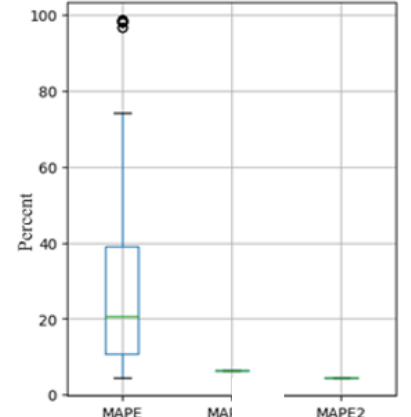

(b)

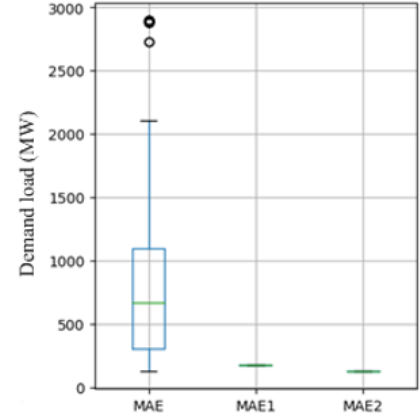

(c)

Figure 4. The box plot of accuracy scores for testing process in case 1: (a) RMSE, (b) MAPE, and (c) MAE 


\subsection{Results of case 2}

In case 2, the training data are for 01 year from 2017-12-04 to 2018-12-02, and the testing data-01 month from 2018-12-03 to 2018-12-30. The results of training process, the optimal ES models and the results of testing process are presented in Table 5. It is revealed that the minimum of AIC and RMSE as well as MAPE and MAE gives the two optimal ES model as indicated in case 2. Figures 5(a) and 5(b) show the series of training and testing process for the first optimal model. The accuracy scores for testing process are plotted in Figure 6.

Table 5. The results of case 2

\begin{tabular}{|c|c|c|c|c|c|c|c|c|}
\hline \multicolumn{4}{|c|}{$\begin{array}{c}\text { The minimum of AIC and accuracy scores of training } \\
\text { process }\end{array}$} & \multirow{2}{*}{$\begin{array}{c}\text { The optimal ES models } \\
(\mathrm{t}, \mathrm{d}, \mathrm{s}, \mathrm{p}, \mathrm{b}, \mathrm{r})\end{array}$} & \multicolumn{4}{|c|}{ The accuracy scores of testing process } \\
\hline $\mathrm{AIC}$ & RMSE & MAPE & MAE & & Accuracy & Optimal & Min & Average \\
\hline \multirow[t]{6}{*}{66648.65} & 44.46909 & & & ['add', True, 'mul', 168, & RMSE & 354.76 & 251.80 & $1,123.99$ \\
\hline & & & & True, True] & MAPE & 10.81 & 7.10 & 23.32 \\
\hline & & & & & MAE & 317.50 & 198.86 & 980.55 \\
\hline & & 1.175906 & 31.87514 & ['add', True, 'mul', 168, & RMSE & 354.77 & 251.80 & $1,123.99$ \\
\hline & & & & True, False] & MAPE & 10.81 & 7.10 & 23.32 \\
\hline & & & & & MAE & 317.51 & 198.86 & 980.55 \\
\hline
\end{tabular}

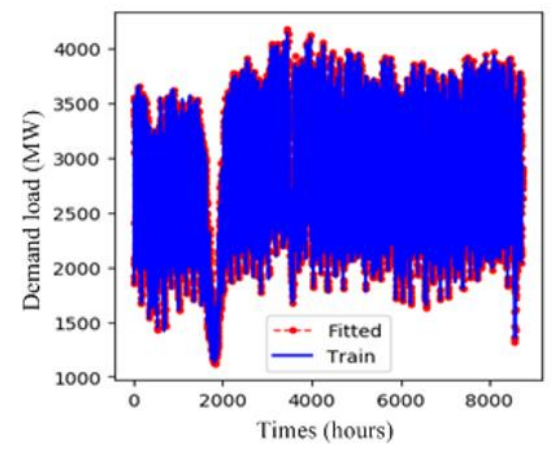

(a)

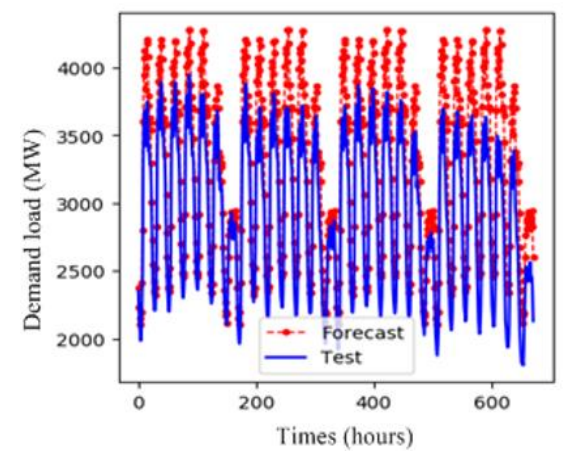

(b)

Figure 5. (a) Fitted and training series, (b) forecast and testing series

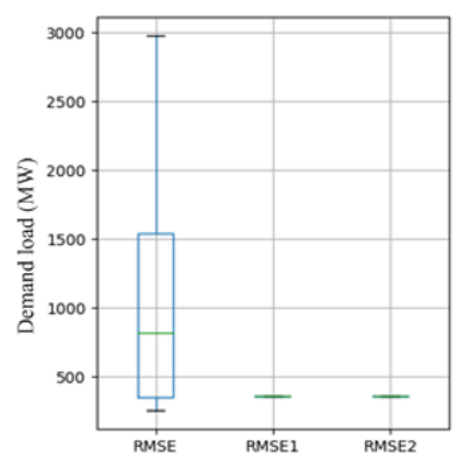

(a)

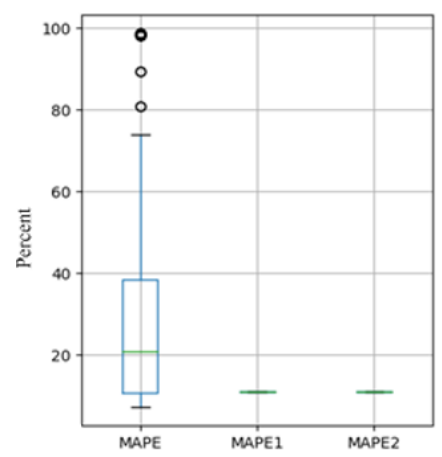

(b)

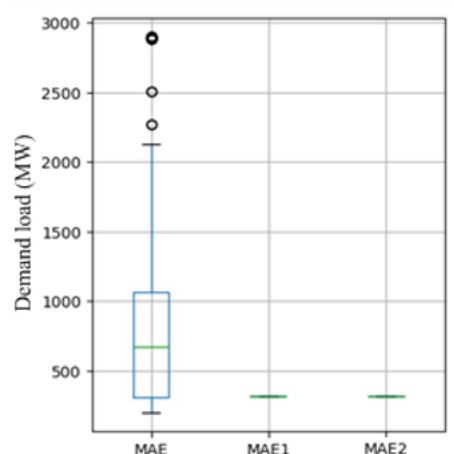

(c)

Figure 6. The box plot of accuracy scores for testing process in case 2: (a) RMSE, (b) MAPE, and (c) MAE

\subsection{Results of case 3}

In case 3, the training data are for 01 week from 2018-12-17 to 2018-12-23, and the testing data-1 week from 2018-12-24 to 2018-12-30. Table 6 shows the results of training process, the optimal ES models and the results of testing process. The minima of AIC, RMSE, MAPE and MAE give the same optimal ES model, so in case 3 we just have 01 optimal model for AIC and accuracy scores. The series for the training and testing processes are presented in Figures 7(a) and 7(b), respectively. Figure 8 shows the box plot of the accuracy scores for testing process. 
Table 6. The results of case 3

\begin{tabular}{|c|c|c|c|c|c|c|c|c|}
\hline \multicolumn{4}{|c|}{$\begin{array}{c}\text { The minimum of AIC and accuracy scores of } \\
\text { training process }\end{array}$} & $\begin{array}{l}\text { The optimal ES } \\
\text { models } \\
(t, d, s, p, b, r)\end{array}$ & \multicolumn{4}{|c|}{ The accuracy scores of testing process } \\
\hline 1281.991 & 38.19976 & 0.903771 & 25.23777 & $\begin{array}{l}\text { ['add', True, 'mul', } \\
\text { 24, True, True] }\end{array}$ & $\begin{array}{c}\text { RMSE } \\
\text { MAPE } \\
\text { MAE }\end{array}$ & $\begin{array}{c}301.79 \\
8.99 \\
252.34\end{array}$ & $\begin{array}{c}298.59 \\
8.86 \\
238.37\end{array}$ & $\begin{array}{c}1,094.01 \\
16.17 \\
945.51\end{array}$ \\
\hline
\end{tabular}

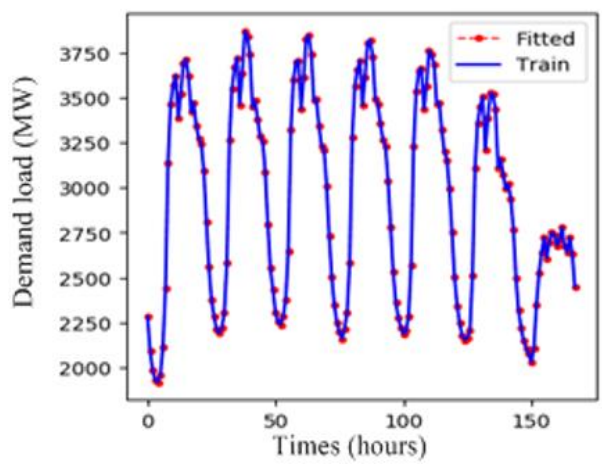

(a)

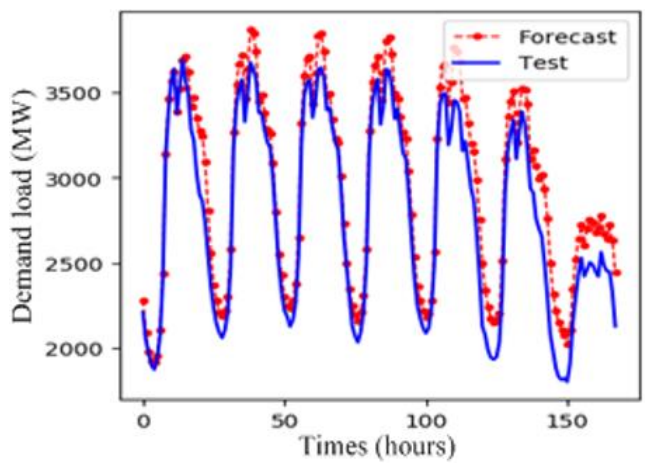

(b)

Figure 7. (a) Fitted and training series, (b) forecast and testing series

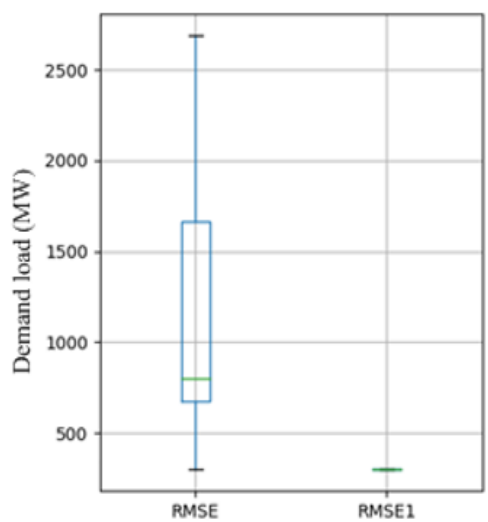

(a)

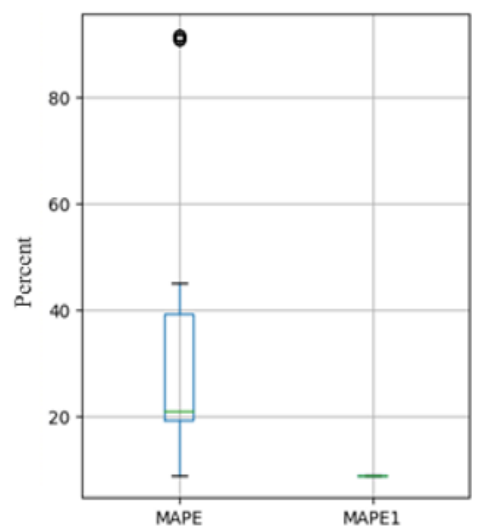

(b)

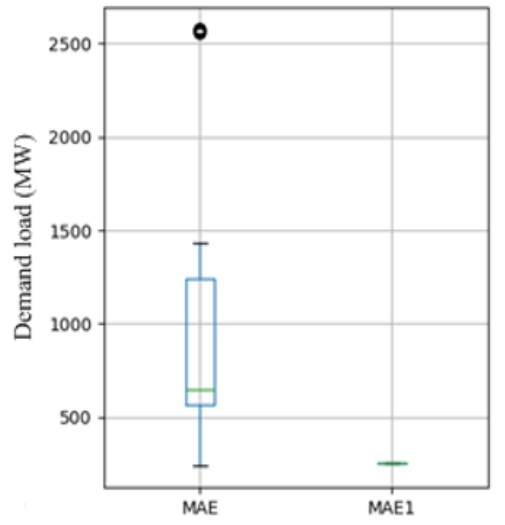

(c)

Figure 8. The box plot of accuracy scores for testing process in case 3: (a) RMSE, (b) MAPE, and (c) MAE

\subsection{Results of case 4}

In case 4, the training data are for 01 month from 2018-11-26 to 2018-12-23, the testing data-1 week from 2018-12-24 to 2018-12-30. Similarly, Table 7 shows the results of training process, the optimal ES models and the results of testing process. The minimum of AIC, RMSE, MAPE and MAE gives the same optimal ES model, indicating the presence of 01 optimal models for AIC and accuracy scores. Figure 9(a), 9(b) show the series for the training and testing processes, respectively. The accuracy scores for testing process built in the box plot are presented in Figure 10.

Table 7. The results of case 4

\begin{tabular}{|c|c|c|c|c|c|c|c|c|}
\hline \multicolumn{4}{|c|}{$\begin{array}{c}\text { The minimum of AIC and accuracy scores of } \\
\text { training process }\end{array}$} & $\begin{array}{l}\text { The optimal ES } \\
\text { models } \\
(t, d, s, p, b, r)\end{array}$ & \multicolumn{4}{|c|}{ The accuracy scores of testing process } \\
\hline 1281.991 & 38.19976 & 0.903771 & 25.23777 & $\begin{array}{c}\text { ['add', True, 'mul', } \\
\text { 24, True, True] }\end{array}$ & $\begin{array}{l}\text { RMSE } \\
\text { MAPE } \\
\text { MAE }\end{array}$ & $\begin{array}{c}301.79 \\
8.99 \\
252.34\end{array}$ & $\begin{array}{c}298.59 \\
8.86 \\
238.37\end{array}$ & $\begin{array}{c}1,094.01 \\
16.17 \\
945.51\end{array}$ \\
\hline
\end{tabular}




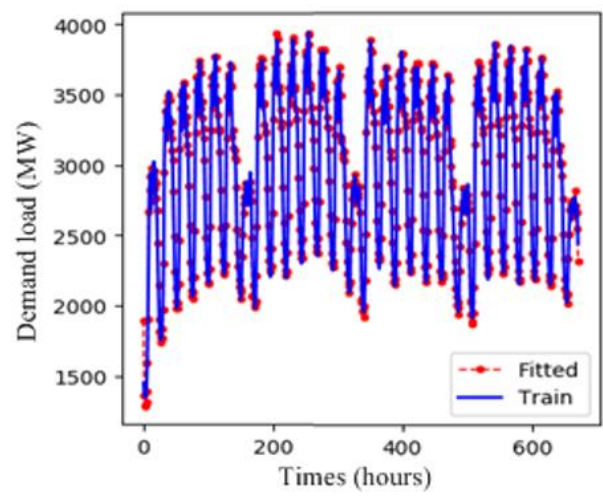

(a)

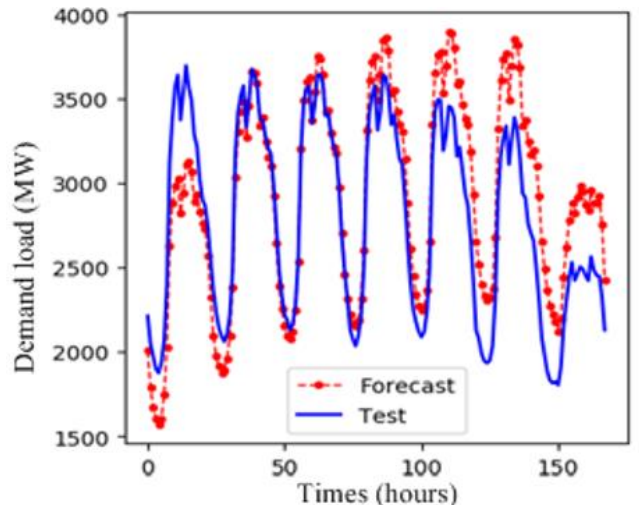

(b)

Figure 9. (a) Fitted and training series, (b) forecast and testing series

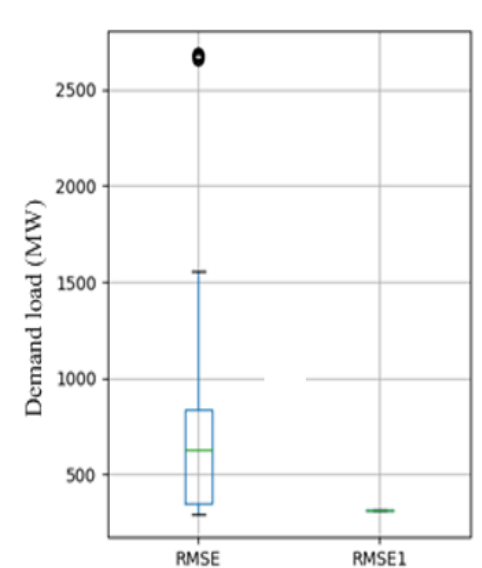

(a)

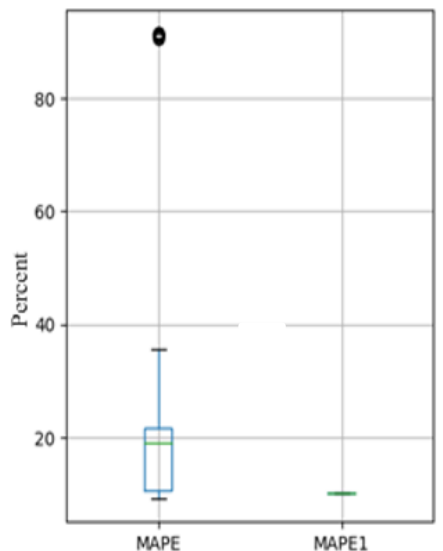

(b)

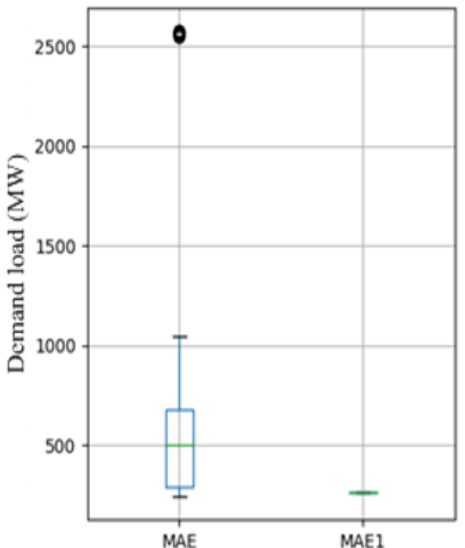

(c)

Figure 10. The box plot of accuracy scores for testing process in case 4: (a) RMSE, (b) MAPE, and (c) MAE

\subsection{Analysis}

In the paper as described above, the optimal of ES models in training process was determined by the minimum of Information Criterion AIC and accuracy scores such as RMSE, MAPE and MAE. In other words, the criterion used to specify the optimal of ES models in training process is this minimum. The analysis of data listed from Table 4 to Table 7 indicated the existence of the optimal model that satisfies the criterion of minimum of Information criterion AIC, accuracy scores RMSE, MAPE, MAE. In cases 1, 2, there are the duplicate optimal models that satisfy both requirements. Especially, in cases 3, 4, there is only one optimal model satisfy all minima of AIC, RMSE, MAPE and MAE.

Noted that the time horizon of training and testing are different in cases 1 to 4 . Indeed, case 1 and case 2 have the same testing time ( 1 month) and different training time ( 1 month and 1 year), case 1 and case 4 have different testing time ( 1 month and 1 week) and the same training time (1 month). The comparison between the ES models described in cases 1 to 4 shows that each time horizon for training and testing has different type of trend and seasonality component from those of other ones. The results showed good values that are promising for applying ES model in different time horizon.

The optimal model obtained in the training process does not ensure completely the optimal results in the testing process. Let's analysis the Table 4 of case 1 for the first optimal model (minimum of AIC and RMSE). When using the first optimal model for the testing process, the accuracy scores of RMSE, MAPE, MAE correspond to 215.24MW, 6.37\%, 177.63 MW, while there are the other ES models that give better results with minimum values of the RMSE, MAPE, MAE are 173.93MW, 4.42\%, 124.88MW, respectively. But in comparison with the average values of all the models (the RMSE, MAPE, MAE values of 1,101.63MW, 24.46\%, 950.33MW, respectively), the accuracy scores of the first optimal model are too small. Besides, analyzing the boxplot of accuracy scores shown in Figure 4 clearly indicates that the accuracy scores of the first optimal model nearly the 
same of the minimum values of all the other models. Moreover, Figure $3 \mathrm{~b}$ also shows the forecast values are well consistent with the testing values for the first optimal model. We have the same results for the second-optimal model (minimum of MAPE, MAE) for the case 1, and the same for case 2, 3, 4. Especially, we have very good results in case 3 and case 4 , in that, we just have only optimal model for each case, and the accuracy scores of the optimal models have nearly the same values with the min values for testing process.

\section{CONCLUSION}

Based on the math formulas of ES taxonomy, a framework for Grid search of ES method was proposed. In the training process, the minimum of AIC, accuracy scores of RMSE, MAPE, MAE was applied to specify the optimal ES models. In the testing process, the accuracy scores have been used to compare the optimal model with all other ones. The load demand data of Ho Chi Minh City were used for the analysis and splitted into many cases to avoid the randomness and to improve the accuracy. The results indicated the existence of the optimal model that satisfies requirement of minimum for Information criterion AIC and accuracy scores. At the same time, in some cases, the optimal model is identified for both of AIC and accuracy scores (case 3,4$)$. In the testing process, the accuracy scores of the optimal model gave the good values close to the minima which were much smaller than the average values as compared to those of all other models. Overall, by verifying the accuracy and reliability of the grid search framework using load demand data in Ho Chi Minh City, the study suggests an effective way for load demand forecasting

\section{REFERENCES}

[1] J. W. Taylor, P. E. McSharry, "Short-term load forecasting methods: an evaluation based on European data," IEEE Transactions on Power Systems, vol. 22, no. 4, pp. 2213-2219, 2007.

[2] P. E. McSharry, S. Bouwman, G. Bloemhof, "Probabilistic forecasts of the magnitude and timing of peak electricity demand," IEEE Transactions on Power Systems, vol. 20, no. 2, pp. 1166-1172, 2005.

[3] E. Gonzalez-Romera, M. A. Jaramillo-Moran, D. Carmona-Fernandez, "Monthly electric energy demand forecasting based on trend extraction," IEEE Transactions on Power Systems, vol. 21, no. 4, pp. 1946-1953, 2006.

[4] M. P. Garcia, D. S. Kirschen, "Forecasting system imbalance volumes in competitive electricity markets," IEEE PES Power Systems Conference and Exposition, 2004, New York, NY, vol. 3, pp. 1805-1812, 2004.

[5] J. W. Taylor, "Density forecasting for the efficient balancing of the generation and consumption of electricity," International Journal of Forecasting, vol. 22, no. 4, pp. 707-724, 2006.

[6] H. L. Imam, M. S. Gaya, and G. S. M. Galadanci, "Short term load forecast of Kano zone using artificial intelligent techniques," Indonesian Journal of Electrical Engineering and Computer Science, vol. 16, no. 2, pp. 562-567, 2019.

[7] K. Kavanagh and K. Kavanagh, "Short Term Demand Forecasting for the Integrated Electricity Market," Student Journal of Energy Research, vol. 2, no. 1, 2017.

[8] Rob J. Hyndman, Anne B. Koehler, J. Keith Ord and Ralph D. Snyder, "Forecasting with Exponential Smoothing The State Space Approach," Springer-Verlag Berlin Heidelberg, 2008.

[9] N. A. Abd Jalil, M. H. Ahmad, and N. Mohamed, "Electricity load demand forecasting using exponential smoothing methods, " World Applied Sciences Journal, vol. 22, no. 11, pp. 1540-1543, 2013.

[10] S. M. Badran, "Short term electrical load forecasting using holt-winters method," Australian Journal of Basic and Applied Sciences (AJBAS), vol. 3, no. 3, pp. 2697-2705, 2009.

[11] H. R. Makridakis S, Wheelwright SC, "Forecasting Methods And Applications," Wiley India Pvt. Limited, pp. 1-632, 2008.

[12] Hyndman, R. J. \& Athanasopoulos, G., "Forecasting: principles and practice," 2019. [Online]. Available: OTexts.org/fpp/.

[13] Jason Brownlee, "Deep Learning for Time Series Forecasting," [Ebook], 2019

[14] J. W. Taylor, "Short-term electricity demand forecasting using double seasonal exponential smoothing," Journal of the Operational Research Society, vol. 54, no. 8, pp. 799-805, 2003.

[15] Taylor W. James, "Exponential smoothing with a damped multiplicative trend," International Journal of Forecasting., vol. 19, no. 4, pp. 715-725, 2003.

[16] B. Billah, M. L. King, R. D. Snyder, and A. B. Koehler, "Exponential smoothing model selection for forecasting," International Journal of Forecasting, vol. 22, no. 2, pp. 239-247, 2006.

[17] R. C. Souza and C. V. C. De Miranda, "Short term load forecasting using double seasonal exponential smoothing and interventions to account for holidays and temperature effects," TLAIO II-2o Taller Lat. Iberoam. Investig. Operaciones, pp. 1-8, 2007.

[18] R. Bindiu, M. Chindriú, and G. V. Pop, "Day-Ahead Load Forecasting Using Exponential Smoothing," Scientific Bulletin of the Petru Maior University of Tirgu Mures, vol. 6, no. 23, pp. 89-93, 2009.

[19] F. Abd. Razak, M. Shitan, A. H. Hashim, and I. Z. Abidin, "Load Forecasting Using Time Series Models," Jurnal Kejuruteraan, vol. 21, no. 1, pp. 53-62, 2009.

[20] S. Kolassa, "Combining exponential smoothing forecasts using Akaike weights," International Journal of Forecasting, vol. 27, no. 2, pp. 238-251, 2011.

[21] G. H. S. Al-maamary, "Short and Medium Iraqi Load Forecast Using Holt-Winter Method And Wavelet Transformation," Canadian Journal on Electrical and Electronics Engineering, vol. 3, no. 5, pp. 225-228, 2012.

[22] T. Jónsson, P. Pinson, H. A. Nielsen, and H. Madsen, "Exponential smoothing approaches for prediction in realtime electricity markets," Energies, vol. 7, no. 6, pp. 3710-3732, 2014.

Grid search of exponential smoothing method: a case study of Ho Chi Minh City load... (Tran Thanh Ngoc) 
[23] B. A. Hoverstad, A. Tidemann, H. Langseth, and P. Ozturk, "Short-Term Load Forecasting With Seasonal Decomposition Using Evolution for Parameter Tuning," IEEE Transactions on Smart Grid, vol. 6, no. 4, pp. 1904-1913, 2015.

[24] B. Liu, J. Nowotarski, T. Hong, and R. Weron, "Probabilistic Load Forecasting via Quantile Regression Averaging on Sister Forecasts," IEEE Transactions on Smart Grid, vol. 8, no. 2, pp. 730-737, 2017.

[25] T. H. Dang-Ha, F. M. Bianchi, and R. Olsson, "Local short term electricity load forecasting: Automatic approaches," Proc. Int. Jt. Conf. Neural Networks, pp. 4267-4274, 2017.

[26] K. Gajowniczek and T. Zabkowski, "Two-stage electricity demand modeling using machine learning algorithms," Energies, vol. 10, no. 10, pp. 1547-1572, 2017.

[27] "Statsmodels.tsa.holtwinters.ExponentialSmoothing," [Online]. 2019. Available: https://www.statsmodels.org/dev/generated/statsmodels.tsa.holtwinters.ExponentialSmoothing.html.

[28] N. Z. Mohd Safar, A. A. Ramli, H. Mahdin, D. Ndzi, and K. M. N. Ku Khalif, "Rain prediction using fuzzy rule based system in north-west Malaysia," Indonesian Journal of Electrical Engineering and Computer Science, vol. 14, no. 3, pp. 1572-1581, 2019.

[29] L. C. Kho, M. S. M. Kasihmuddin, M. A. Mansor, and S. Sathasivam, "Logic mining in football matches," Indonesian Journal of Electrical Engineering and Computer Science, vol. 17, no. 2, pp. 1074-1083, 2019.

[30] R. Meenal, A. Immanuel Selvakumar, and E. Rajasekaran, "Review on mathematical models for the prediction of solar radiation," Indonesian Journal of Electrical Engineering and Computer Science, vol. 15, no. 1, pp. 54-59, 2019.

[31] S. R. M-Dawam and K. R. Ku-Mahamud, "Reservoir water level forecasting using normalization and multiple regression," Indonesian Journal of Electrical Engineering and Computer Science, vol. 14, no. 1, pp. 443-449, 2019.

\section{BIOGRAPHIES OF AUTHORS}

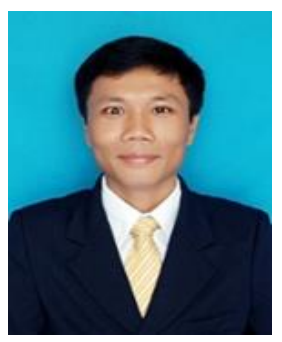

Tran Thanh Ngoc received the B.Sc. and M.Sc. degrees from the Ho Chi Minh University of Technology, Vietnam in 2001 and 2006, respectively. He received his Ph.D. degree in Information and electricity engineering from Hunan University, Changsha, China in 2012. Currently, he is a Lecturer with the Faculty of Electrical Engineering Technology, Industry University of Ho Chi Minh City, Vietnam. His main research interests include HVDC transmission systems, active filters, power quality and load forecasting

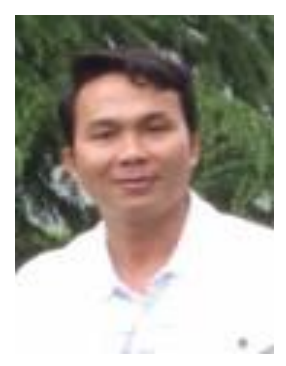

Le Van Dai received MSc degree in Electrical Engineering from the Ho Chi Minh City University of Technology, Vietnam in 2008. He received his Ph.D. degree in Information and electricity engineering from Hunan University, Changsha, China in 2016. Since 2011, he is a lecturer of the Faculty of Electrical Engineering Technology, Industry University of Ho Chi Minh City, Vietnam. His research interests include FACTS control, solar PV energy conversion, control of wind power generation, and grid integration 